\title{
Tandem Mass Spectroscopy in Diagnosis and Clinical Research
}

\author{
Rama Devi Mittal ${ }^{1}$
}

Published online: 4 April 2015

(C) Association of Clinical Biochemists of India 2015

Tandem mass spectrometry, also known as MS/MS or $\mathrm{MS}^{2}$, involves multiple steps of mass spectrometry selection, with some form of fragmentation occurring in between the stages. Mass spectrometry is a powerful technique for chemical analysis that is used to identify unknown compounds, to quantify known compounds, and to elucidate molecular structure. To understand the principle of operation it is established that a mass spectrometer is a "molecule smasher" that measures molecular and atomic masses of whole molecules, molecular fragments and atoms by generation and detection of the corresponding gas phase ions, separated according to their mass-to-charge ratio $(\mathrm{m} / \mathrm{z})$. It measures masses correspond to molecular structure and atomic composition of parent molecule and hence allows determination and elucidation of molecular structure [1].

Now the pertinent question comes to mind that why mass spectrometry? It may also be used for quantitation of molecular species. It is considered a very sensitive technique and works with minute quantities of samples (as low as $10^{-12} \mathrm{~g}, 10^{-15} \mathrm{~mol}$ ) and is easily interfaced with chromatographic separation methods for identification of components in a mixture. Mass spectrometry also provides valuable information to a wide range of professionals: chemists, biologists, physicians, astronomers, environmental health specialists. It works by generating spectrum by separating ions of different mass to charge ratio $(\mathrm{m} / \mathrm{z})$ where $\mathrm{m}$ is the molecular or atomic mass, $\mathrm{z}$ is the electrostatic charge unit. In many cases (such as small

Rama Devi Mittal

ramamittal@gmail.com

1 Department of Urology and Renal Transplantation, Sanjay Gandhi Post Graduate Institute of Medical Sciences, Raebareli Road, Lucknow 226014, Uttar Pradesh, India molecules), $\mathrm{z}=1$ measured $\mathrm{m} / \mathrm{z}=$ mass of fragment. But this is not always true for large bio-molecules analyzed by electro spray (ESI), z > 1 [2].

Tandem mass spectrometer is of many different typeseach has different advantages, draw-backs and applications. All consist of four major sections linked together inlet-ionization source-analyser-detector. All sections are usually maintained under high vacuum and the functions of instrument control, sample acquisition and data processing are under computer control. Data system and computer control is often overlooked-most significant advance in mass spectrometry-allows 24/7 automation and development of modern powerful analytical techniques [3].

Tandem mass spectrometer is a single instrument using two (or more) mass analyzers. Simplest form consists of two mass spectrometers (MS/MS) in series connected by a chamber known as a collision cell. The sample to be examined is essentially sorted and weighed in the first mass spectrometer, then broken into pieces in the collision cell, and a piece or pieces sorted and weighed in the second mass spectrometer. The tandem mass spectrometer is constructed of two or more quadrupoles, with a collision cell separating each quadrupole. Once a sample has been separated by chromatography, the substances initially go through an initial quadrupole which separates the mixture of ions allotting only certain ions (precursor ions), passage to the collision cell. The first quadrupole is used to select user-specified sample ions from a specific component; usually the molecular-related [i.e. $(\mathrm{M}+\mathrm{H})+$ or $(\mathrm{M}-\mathrm{H})^{-}$] ions within the collision cell the precursor-ions also known as "parent ions" are then bombarded with an inert gas (Xe, Ar, etc.) and are further broken down into different charged and mass ions (product ions). These product ions also known as "daughter ions" are then run through an additional quadrupole to further separate the ions which is set to monitor specific ion 
fragments. This process can be repeated several times in order to get highly specific readings [4]. There are several applications of tandem mass spectrometer. Clinical testing and toxicology, inborn errors of metabolism-newborn screening, cancer, diabetes, various poisons, drugs of abuse, etc. Biotechnology and pharmaceutical to determine chemical structure of drugs and drug metabolites, detection/ quantification of impurities, drugs and their metabolites in biological fluids and tissues. High through-put drug screening, analysis of liquid mixtures, fingerprinting, nutraceuticals/herbal drugs/tracing source of natural products or drugs and many more. Protein sequencing and identification protein Identification via database search (SPC and spectral alignment), de novo peptide sequencing (spectrum graph), hybrid, identifying post translationally modified (PTM) peptides quantitative proteomics, identifying proteins that are differentially abundant, besides it has a significant role in proteomics [5, 6].

\section{Mass Spectrometry Analysis in New Born Screening Programs}

There are three groupings of disorders. Aminoacidopathies are detected using a methodology referred to as a neutral loss assay for specific amino acids in the sample. Organic acidurias and fatty acid oxidation disorders a full acylcarnitine profile is performed to detect fatty acid oxidation disorders and organic acidurias. Aminoacidopathies MS/MS technology allows for improvement in and consolidation of methods for detecting amino acid disorders citrullinemia maple syrup urine disease, homocystinuria phenylketonuria argininosuccinic aciduria tyrosinemia MS/MS technology allows for improvement in and consolidation of methods for detecting amino acid disorders. Thus, along with MSUD, PKU and homocystinuria, state programs can now test for argininosuccinic aciduria, citrullinemia and tyrosinemia [7].

\section{Expanded New Born Screening}

In the past, this technology was time-consuming and expensive. Both of these factors (time and cost) made MS/MS impractical for newborn screening. Newborn screening requires a large number of tests to be done quickly, accurately and inexpensively. It was only after MS/MS became automated that it became possible to use this technology in newborn screening. Expanded newborn screening uses a new technology called tandem mass spectrometry (MS/MS) to screen newborns for over 30 disorders. Only a few drops of the baby's blood are needed to do this expanded newborn screening test. Expanded newborn screening is also fast, accurate, and cheap. This identifies and measures two different groups of substances—acylcarnitines and amino acids. Using MS/MS, the lab will measure which acylcarnitines and amino acids are present in the newborn's blood. It will also measure how much of each substance is present in the newborn's blood. In this way, MS/MS can be used to screen for over thirty different rare inherited metabolic disorders.

\section{False Positives}

The importance of reducing the false positive rate is obvious, as it leads to unnecessary familial anxiety as well as increased costs in the work-up. Tandem mass spectrometer is estimated to reduce the false positive rate seen with traditional testing methodologies from about 1.5 to about $0.26 \%$. However, it is important to keep in mind that there are still false positive results. The diseases natural history also impacts the false negative rate with variable conditions not being detected in the newborn period if they have a milder clinical course.

\section{Direct Multiplex Assay of Lysosomal Enzymes in Dried Blood Spots for Newborn Screening}

Newborn screening for deficiency in the lysosomal enzymes that cause Fabry, Gaucher, Krabbe, Niemann-Pick $\mathrm{A} / \mathrm{B}$, and Pompe diseases is warranted because treatment for these syndromes is now available or anticipated in the near feature. Wagner et al. described a multiplex screening method for all five lysosomal enzymes that uses newbornscreening cards containing dried blood spots as the enzyme source [8]. Analyzed dried blood spots from 5 patients with Gaucher, 5 with Niemann-Pick A/B, 11 with Pompe, 5 with Fabry, and 12 with Krabbe disease, and in all cases the enzyme activities were below the minimum activities measured in a collection of heterozygous carriers and healthy noncarrier individuals. The enzyme activities measured in five to nine heterozygous carriers were approximately one-half those measured with 15-32 healthy individuals, but there was partial overlap of each condition between the data sets for carriers and healthy individuals. For all five diseases, the affected individuals were detected. The assay can be readily automated, and the anticipated reagent and supply costs are well within the budget limits.

\section{Metabolomics Research with Tandem Mass Spectrometry}

Metabolomics, the large-scale analysis of metabolites generated in metabolic pathways, has recently joined with "omics" integration studies. Various state of the art 
techniques have been applied to metabolomics studies, including nuclear magnetic resonance (NMR) spectroscopy, magnetic resonance imaging (MRI), infrared spectroscopy (IR), gas chromatography mass spectrometry (GC-MS), capillary electrophoresis-MS and HPLC-MS. The tandem mass spectrometry is a vital technique in identifying and quantifying different metabolites [8]. The targeted metabolomics experiment with tandem mass spectrometry measures defined ion transitions from known metabolites. The untargeted metabolomics experiment records all ions within a certain mass range, including the ions belonging to structurally novel metabolites [9].

Therapeutic drug monitoring of immunosuppressant drugs (ISDs) is well established. The importance of monitoring these drugs is due to their narrow therapeutic window. Elevated dosing of the ISDs can cause significant toxicity while under dosing can result in transplant rejection. Because of this narrow therapeutic window, the ISDs are considered critical dose drugs, requiring individualized drug therapy by measuring the actual drug concentrations in each patient to maximize the therapeutic response and minimize adverse side effects $[10,11]$.

Genome-wide association scans with high-throughput metabolic profiling provide unprecedented insights into how genetic variation influences metabolism and complex disease. Here, they reported the most comprehensive exploration of genetic loci influencing human metabolism thus far, comprising 7824 adult individuals from two European population studies. Researchers reported genome-wide significant associations at 145 metabolic loci and their biochemical connectivity with more than 400 metabolites in human blood. They extensively characterized the resulting in vivo blueprint of metabolism in human blood by integrating it with information on gene expression, heritability and overlap with known loci for complex disorders, inborn errors of metabolism and pharmacological targets [12].

\section{Summary}

The implementation of mass spectrometry approaches in the clinical laboratory has resulted in significant advancements in clinical pathology while adding a new layer of complexity to an already overwhelming aspect of medicine. MS/MS can provide substantial benefits to patients and their families if thoughtfully integrated into newborn screening programs. Mass spectrometers will be the key technology in Therapeutic drug monitoring, in proteomics as well as metabolomics, to advance our understanding of disease biology and deliver new bio. The use of tandem mass spectrometry is well documented in the clinical laboratory. In particular, the development of atmospheric pressure ionization techniques to perform/MS has allowed the clinical chemist to address new areas of research and drastically improve the quality of clinical assays due to higher specificity and better sensitivity than other approaches.

\section{References}

1. Jeevan KP. Tandem mass spectrometry: applications and principles. Biochemistry, genetics and molecular biology. Rijeka: InTech; 2012.

2. Frese CK, Altelaar AF, van den Toorn H, Nolting D, GriepRaming J, Heck AJ, et al. Toward full peptide sequence coverage by dual fragmentation combining electron-transfer and higherenergy collision dissociation tandem mass spectrometry. Anal Chem. 2012;84:9668-73.

3. Oliveira RV, Henion J, Wickremsinhe ER. Automated high-capacity on-line extraction and bioanalysis of dried blood spot samples using liquid chromatography/high-resolution accurate mass spectrometry. Rapid Commun Mass Spectrom. 2014;28: 2415-26.

4. Dittrich J, Becker S, Hecht M, Ceglarek U. Sample preparation strategies for targeted proteomics via proteotypic peptides in human blood using liquid chromatography tandem mass spectrometry. Proteomics Clin Appl. 2015;9:5-16.

5. Angel TE, Aryal UK, Hengel SM, Baker ES, Kelly RT, Robinson EW, et al. Mass spectrometry-based proteomics: existing capabilities and future directions. Chem Soc Rev. 2012;41:3912-28.

6. Chapman JD, Goodlett DR, Masselon CD. Multiplexed and dataindependent tandem mass spectrometry for global proteome profiling. Mass Spectrom Rev. 2014;33:452-70.

7. Tomatsu S, Fujii T, Fukushi M, Oguma T, Shimada T, Maeda M, et al. Newborn screening and diagnosis of mucopolysaccharidoses. Mol Genet Metab. 2013;110:42-53.

8. Tonoli D, Varesio E, Hopfgartner G. The use of mass spectrometry to analyze dried blood spots. Spectrom Rev. 2014. doi:10.1002/mas.21441.

9. Gieger C, Geistlinger L, Altmaier E, Hrabé de Angelis M, Kronenberg F, Meitinger T, et al. Genetics meets metabolomics: a genome-wide association study of metabolite profiles in human serum. PLoS Genet. 2008;4:e1000282.

10. Yang Z, Wang S. Recent development in application of high performance liquid chromatography-tandem mass spectrometry in therapeutic drug monitoring of immunosuppressants. J Immunol Methods. 2008;336:98-103.

11. Meinitzer A, Gartner G, Pilz S, Stettin M. Ultra fast liquid chromatography-tandem mass spectrometry routine method for simultaneous determination of cyclosporin A, tacrolimus, sirolimus, and everolimus in whole blood using deuterated internal standards for cyclosporin A and everolimus. Ther Drug Monit. 2010;32:61-6.

12. Illig T, Gieger C, Zhai G, Römisch-Margl W, Wang-Sattler R, Prehn C, et al. A genome-wide perspective of genetic variation in human metabolism. Nat Genet. 2010;42:137-41. 